\title{
Russian Experience of the Rational Thromboprophylaxis in Traumatology and Orthopaedics
}

\author{
Firsov Sergey Anatolyevich, Levshin Andrey and Matveev Rudolf \\ Non-governmental Healthcare Institution "Yaroslavl Railway Clinical Hospital”, Joint Stock Company “Russian Railways”, Center \\ of Traumatology and Orthopaedics, Yaroslavl, State budgetary educational institution of Higher Professional Education Northern \\ State Medical University, Arkhangelsk 150000, Russian Federation
}

\begin{abstract}
Analysis of the safety and efficacy of the currently marked oral anticoagulants was performed in patients after large joints arthroplasty. A total of 5025 patients after total knee arthroplasty and 5216 patients after total hip arthroplasty were examined. All patients were divided into groups by the prescribed anticoagulant. The duration of anticoagulant therapy was 35 days and 6 weeks after surgery, respectively. Ultrasonography of the lower extremities veins was performed in all patients prior to the surgery and 5 days after surgery. In patients receiving Dabigatran, the incidence of clinically significant deep vein thrombosis was lower compared with patients receiving Rivaroxaban and Apixaban; in contrast, the rate of postoperative hematomas in the last two groups was higher.
\end{abstract}

Key words: Thromboprophylaxis, arthroplasty, oral anticoagulants.

\section{Introduction}

The development of venous thrombosis and thromboembolism in traumatology and orthopedics is still a huge issue despite the available standards and clinical guidelines. Data from clinical studies, both national and foreign, suggest that the primary prevention of venous thrombosis may significantly reduce the incidence of deep vein thrombosis and $\mathrm{PE}$ (pulmonary embolism) [1-3]. In Europe, the annual number of deaths after venous thromboembolism reaches 600,000 cases. In comparison, breast cancer kills 86,831 people annually, prostate cancer-63,636, traffic accidents - 53,599, and AIDS - 5,860 people [4].

Knee and hip joints surgery is an intervention with high risk of thrombosis and thromboembolism; the incidence of thrombotic events without anticoagulant therapy may reach 57 and 85\%, respectively [4-8]. PE (pulmonary embolism) is a particularly severe complication; its incidence may reach $28 \%$ [7, 8].

Corresponding author: Firsov Sergey Anatolyevich, Doctor of Medical Science, Head of the Centre of Traumatology and Orthopaedics.
Anticoagulant therapy may reduce incidence of these complications tenfold. Moreover, if the patient has additional risk factors, deep vein thrombosis may complicate the postoperative period even after minimally invasive arthroscopic surgery $[2,9,10]$.

Currently, the wide range of anticoagulant drugs are marketed [1]. However, orthopedic and traumatic surgeon is still concerned about the optimal balance between effective prevention of the deep vein thrombosis and the risk of major bleeding [10, 11]. All marketed drugs are excellent anticoagulants; however, all of them have adverse effects, and bleeding in the postoperative period is the most dangerous. The risk of these adverse events is in particularly determined by the characteristics of the patient and the drug pharmacokinetics.

Data from many clinical studies show that the efficacy and safety of thromboprophylaxis is not significantly depend on the time of the therapy initiation [11, 12]. Comparative results of the first dose effect of the anticoagulant administered 12 hours and 2 hours before the surgery show that anticoagulant administration 2 hours before the surgery affects only 
the severity of the intraoperative hemorrhage towards its increase $[7,9,13]$. Therefore, many early clinical guidelines suggested only starting anticoagulant therapy after surgery, and the time of the therapy initiation depended on the particular drug.

Objective of the study was to evaluate the safety and efficacy of the oral anticoagulants in the national pharmaceutical market in patients undergoing large joints arthroplasty.

\section{Patients and Methods}

We performed the analysis of the case histories of patients who were treated at Russian hospitals after total hip arthroplasty and total knee arthroplasty and were receiving thromboprophylaxis with oral anticoagulants since 2009 to 2014 . The number of patients after TKAP (total knee arthroplasty) was 5025; the number of patients after THAP (total hip arthroplasty) was 5216. Age of the patients was 55.4 years (95\% CI (confidence interval) 35-75).

All patients were divided into groups by the administered anticoagulant drug. Dabigatran etexilate was administered at dose of $220 \mathrm{mg}$ once daily, rivaroxaban was administered at dose of $10 \mathrm{mg}$ once daily, and apixaban (starting since 2013) was administered at dose of $2.5 \mathrm{mg}$ twice daily (Table 1). The duration of treatment with these agents was 35 days after surgery in patients with THAP and 6 weeks after surgery in patients with TKAP * (see page 6). According to clinical guidelines, dabigatran was started 4 hours after the surgery, rivaroxaban - 10 hours after the surgery, and apixaban - 12 hours after the surgery.

In addition, all patients had additional thromboprophylaxis with intermittent compression device and compression stockings.
All patients underwent twice Doppler ultrasound of the lower limb veins: before surgery and before discharge from the hospital on the fifth postoperative day.

The safety of anticoagulant drugs was assessed based on the incidence of bleeding in the postoperative period; the effectiveness was assessed based on the incidence of clinically significant deep vein thrombosis of the lower limbs.

Statistical analysis was performed using EpiInfo (TM) software v. 3.4.1 and SPSS 17.0 for Windows. Quantitative data are presented as the arithmetic mean. Nominal data are presented as relative frequencies and 95\% confidence intervals. Results were considered significant at $\mathrm{p}<0.05$.

\section{Results}

Among patients after TKAP, deep vein thrombosis of the lower extremities was less frequent in the group of patients receiving dabigatran-214 (9\%) patients (Table 2). The highest incidence was recorded in the apixaban group -93 (15.2\%) patients. Postoperative hematomas were also less frequent in the dabigatran group $-2.1 \%$ of patients; in the rivaroxaban group this adverse event was reported in $5.8 \%$ of patients $(p<$ $0.05)$, in the apixaban group - in $5.4 \%$ of patients $(\mathrm{p}<$ $0.05)$. Fatal pulmonary embolism occurred only in dabigatran the group (3 $(0.1 \%)$ patients), but the difference with the other groups was not significant. The incidence of non-fatal pulmonary embolism in all three study groups was comparable (see Table 2). The incidence of asymptomatic venous thrombosis (diagnosed by ultrasound at day 5 after surgery) was comparable in the dabigatran and rivaroxaban groups noted - 11 and $11.8 \%$, respectively, whereas in the apixaban group the incidence of asymptomatic venous

Table 1 The distribution of patients by groups.

\begin{tabular}{llll}
\hline Surgery & Dabigatran & Rivaroxaban & Apixaban \\
\hline TKAP $(\mathrm{n}=5025)$ & 2359 & 2054 & 612 \\
THAP $(\mathrm{n}=5216)$ & 2746 & 1945 & 525 \\
Total & 5105 & 3999 & 1137 \\
\hline
\end{tabular}


thrombosis was nearly 2-fold higher, although no statistically significant differences have been identified due to the small the sample size (see Table 2).

In general, a similar trend was found in the patients who received thromboprophylaxis after THAP, (Table 3). Deep vein thrombosis in the dabigatran group was diagnosed in $4.9 \%$ of patients, in the rivaroxaban group - in $8.6 \%$ of patients $(p<0.05)$, in the apixaban group - in $12.9 \%$ of patients. The incidence of postoperative hematoma was significantly $(\mathrm{p}<0.01)$ lower in the group of patients receiving dabigatran $-4.9 \%$ compared to 8.6 and $11.2 \%$ in the rivaroxaban and apixaban groups, respectively. Two $(0.06 \%)$ cases of fatal pulmonary embolism were reported in the dabigatran group (see Table 3).

\section{Discussion}

Requirements to the current anticoagulant drugs, are the follows: the drug should provide high efficacy along with a low risk of bleeding, ease of use (oral, no need for dosage adjustments and special monitoring), safety (may be administered in elderly patients with renal insufficiency, hepatic insufficiency) and convenient for use in ambulatory practice [14-17].

Dabigatran, rivaroxaban, and apixaban are the new oral anticoagulant drugs widely used in traumatology and orthopedics in the management of patients undergoing hip and knee arthroplasty [4-6]. These anticoagulants are claimed as effective, convenient and safe agents. They require no continuous laboratory monitoring and dose titration as warfarin. Their undoubted advantage is lack of thrombocytopenia during long-term treatment in contrast to heparin. However, clinical studies of their safety and efficacy in traumatology and orthopedics and continuing at present time [18].

In 2010, M. Huisman et al. [13] summarized the data on the efficacy and safety of dabigatran and rivaroxaban obtained from the six phase III randomized trials that included 18,450 patients undergoing hip and knee arthroplasty. The efficacy was assessed based on the incidence of venous thromboembolism and overall mortality, the safety was assessed based on the incidence of bleeding.

Table 2 The incidence of thrombotic and bleeding complications in patients after TKAP.

\begin{tabular}{llll}
\hline Complication & Dabigatran & Rivaroxaban & Apixaban \\
\hline Deep vein thrombosis (clinically significant) & $214(9 \%, 95 \%$ & $251 *(12.2 \%, 95 \%$ & $93(15.2 \%, 95 \%$ \\
& CI $6.9-9.1)$ & CI $10.5-13.4)$ & CI $13.7-16.3)$ \\
Postoperative hematoma & $49(2.1 \%, 95 \%$ & $121 *(5.8 \%, 95 \%$ & $33(5.4 \%, 95 \%$ \\
& CI $0.8-3.5)$ & CI $4.2-7.2)$ & CI $4.1-6.8)$ \\
Non-fatal PE & $12(0.4 \%, 95 \%$ & $18(0.9 \%, 95 \%$ & $6(0.8 \%, 95 \%$ \\
& CI $0.1-0.7)$ & CI $0.4-1.4)$ & CI $0.3-1.3)$ \\
Fatal PE & $3(0.1 \%, 95 \%$ & - & - \\
Asymptomatic venous thrombosis & CI $0.05-0.2)$ & $243(11.8 \%, 95 \%$ & $136(22.2 \%, 95 \%$ \\
(diagnosed by ultrasound) & $296(11 \%, 95 \%$ & CI $6.4-12.9)$ & CI $20.8-23.7)$ \\
\hline
\end{tabular}

Note. The significance of differences compared with the dabigatran group: $*$ - $<<0.05, * *-p<0.01$ (Tables 2 and 3 ).

Table 3 The incidence of thrombotic and bleeding complications in patients after THAP.

\begin{tabular}{llll}
\hline Complication & Dabigatran & Rivaroxaban & Apixaban \\
\hline Deep vein thrombosis (clinically significant) & $133(4.9 \%, 95 \%$ & $167 *(8.6 \%, 95 \%$ & $68(12.9 \%, 95 \%$ \\
& CI $2.9-5.4)$ & CI $7.1-9.7)$ & CI $12.4-15.2)$ \\
Postoperative hematoma & $134(4.9 \%$, & $169^{*}(8.6 \%, 95 \%$ & $59 * *(11.2 \%, 95 \%$ \\
& $95 \%$ CI $3-5)$ & CI $8.2-10.2)$ & CI $10.2-12.4)$ \\
Non-fatal PE & $8(0.2 \%, 95 \%$ & $5(0.3 \%, 95 \%$ & $4(0.6 \%, 95 \%$ \\
& CI $0.1-0.3)$ & CI $0.2-0.4)$ & CI $0.3-0.9)$ \\
Fatal PE & $2(0.06 \%, 95 \%$ & - & - \\
Asymptomatic venous thrombosis & CI $0.02-0.09)$ & $256 * *(13.1 \%, 95 \%$ & $69(13.1 \%, 95 \%$ \\
(diagnosed by ultrasound) & $245(8.9 \%, 95 \%$ & CI $12.7-14.9)$ & CI $12.2-15.0)$ \\
\hline
\end{tabular}


Table 4 The incidence of complications (\%) during anticoagulation therapy according to the XAMOS study [20].

\begin{tabular}{llll}
\hline Endpoint & $\begin{array}{l}\text { Rivaroxaban } \\
\left(\mathrm{n}=8548^{3}\right)\end{array}$ & $\begin{array}{l}\text { Standard therapy } \\
\left(\mathrm{n}=7968^{3}\right)\end{array}$ & Relative risk (95\% CI) \\
\hline Major bleedings $^{1}$ & 0.4 & 0.3 & $1.35(0.94-1.93)$ \\
Major bleedings EMA $^{2}$ & 1.9 & 1.5 & $1.21(1.01-1.45)$ \\
Minor bleedings EMA & 4.4 & 2.9 & $1.52(1.35-1.71)$ \\
Minor bleedings & 3 & 1.7 & $1.76(1.52-2.04)$ \\
All bleedings & 4.9 & 3.3 & $1.50(1.34-1.68)$ \\
Wound discharge & 0.5 & 0.2 & $2.65(1.78-3.92)$ \\
\hline
\end{tabular}

Note. '-clinically significant bleeding, fatal or bleeding in a critical organ, or requiring re-operation, or bleeding outside the surgical wound and accompanied by a decrease of hemoglobin level by $2 \mathrm{~g} / \mathrm{dL}$ or more, or to require transfusion of 2 or more blood units. ${ }^{2}$-Major bleedings EMA (European Medicines Agency) - also included bleedings leading to the treatment discontinuation, or bleeding from the surgical wound accompanied by a decrease of hemoglobin level by $2 \mathrm{~g} / \mathrm{dL}$ or more, or to require transfusion of 2 or more blood units. ${ }^{3}$ - presented data are adjusted for safety population.

Dabigatran was comparable to enoxaparin, whereas rivaroxaban was less safe. Thus, the risk of major bleeding during rivaroxaban therapy increased by $21 \%$, and the risk of all bleeding increased by $50 \%$ in comparison with standard anticoagulation therapy.

In 2012, N. Rosencher et al. [19] published data from the international observational clinical study where 5292 patients undergoing hip and knee arthroplasty were treated with dabigatran $220 \mathrm{mg} /$ day as anticoagulant. In $40 \%$ of patients, more than one potential risk factor for bleeding and/or venous thromboembolism was reported. The incidence of major bleeding was $0.72 \%$ (95\% CI, $0.51-0.98 \%)$, the incidence of all bleeding was $3.82 \% \quad(95 \%$ CI, $3.32-4.37 \%$ ). In this study, dabigatran was characterized by a low risk of bleeding even in patients with positive history (chronic heart failure, history of venous thromboembolic events, long-term use of nonsteroidal anti-inflammatory drugs, active smoking, concomitant aspirin therapy, and coronary artery disease).

According to the results of the open-label post-marketing study XAMOS (17,701 patients after THAP or TKAP) published in 2014, rivaroxaban was characterized as a highly effective drug for all thromboembolic events [20]. However, the risk of bleeding complications was increased compared with dabigatran and fondaparinux (Table 4).

A new oral anticoagulant drug apixaban that has relatively recently appeared in the Russian market is different from dabigatran and rivaroxaban. Apixaban is a structurally new neutral bicyclic pyrazole that works as a selective factor Xa inhibitor [21]. In 2010, the first data on this drug from the ADVANCE-3 study that included 1949 patients were published. Apixaban was administered to the patients undergoing total hip and knee arthroplasty. Venous thromboembolic event were reported in $1.4 \%(95 \% \mathrm{CI}, 1.22-1.54)$ of patients in the apixaban group compared with $3.9 \%$ of patients in the enoxaparin group. The incidence of major bleeding was $0.8 \%$, whereas in the enoxaparin group the incidence of major bleeding was $0.7 \%$ (see Figure). No fatal bleeding in either group was reported [21].

In this study, dabigatran etexilate showed the maximum efficiency in preventing clinically significant deep vein thrombosis of the lower extremities after the TKAP (9\% vs. 12.2 and $15.2 \%$ in the rivaroxaban and apixaban groups, respectively), and after THAP (4.9 vs. $8.6 \%$ and $12.9 \%$, respectively).

The incidence of postoperative hematomas in the dabigatran group also was lower, accounting for 2.1 and $4.9 \%$ after TKAP and THAP, respectively. In the rivaroxaban and apixaban groups, this complication was diagnosed almost 2-fold more frequently regardless of the type of surgery.

Comparative data on rivaroxaban and dabigatran are consistent with the results of clinical studies where these drugs were compared with enoxaparin (Table 5).

Thus, data obtained in the present study suggest that 
Table 5 The incidence of clinically significant bleedings during therapy with new anticoagulants compared with enoxaparin [22].

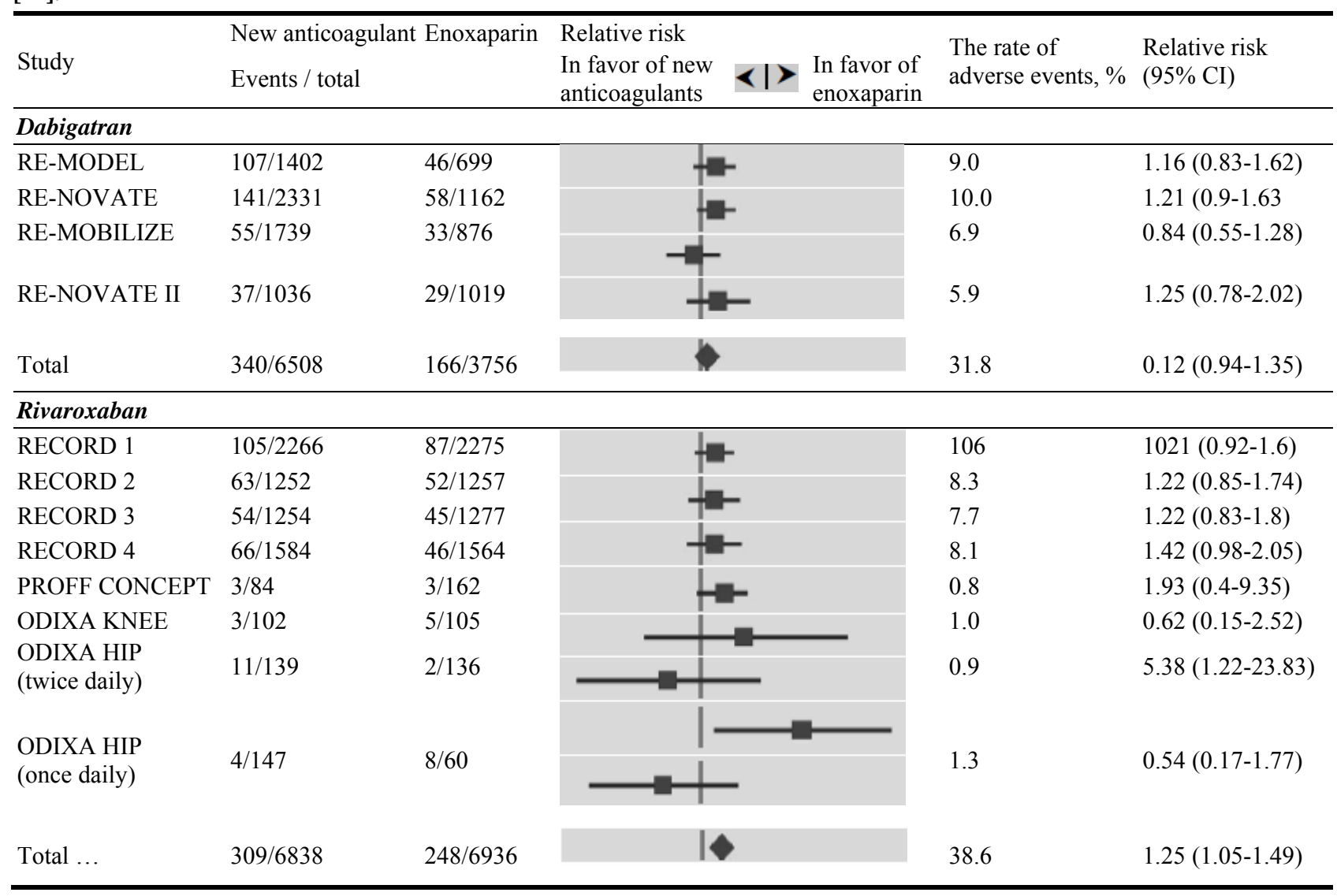

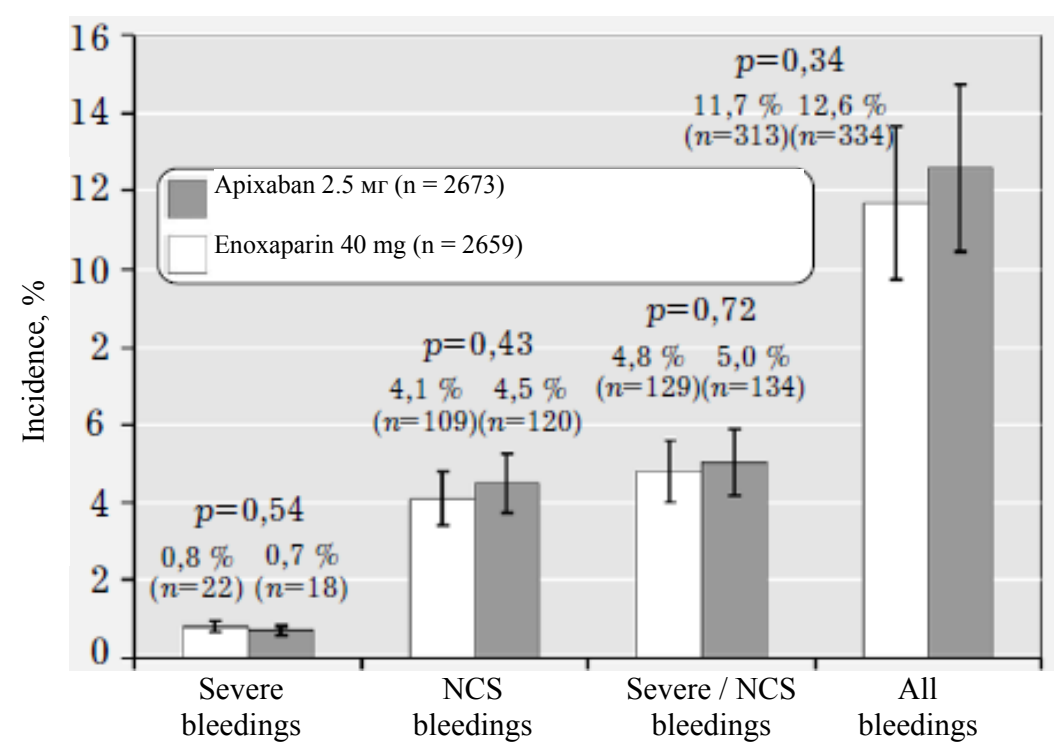

The incidence of bleeding during treatment with anticoagulants according to the ADVANCE-3 study [21].

NCS - Nonsevere clinically significant.

new oral anticoagulant drugs are safe and effective in preventing thrombosis in traumatology and orthopedics. However, treatment with these drugs is associated with the risk of serious adverse events, including postoperative bleeding. Our 6-year experience of the use of these drugs and results of the 
international studies suggest that rivaroxaban demonstrated the worst results in terms of bleeding in the postoperative period. Dabigatran etexilate should be recognized as the drug with the highest efficiency and the best safety profile, as it showed the best results in patients undergoing major orthopedic surgery of the knee and hip joints.

According to the Pradaxa ${ }^{\circledR}$ prescribing information, for the prevention of venous thromboembolism after total knee arthroplasty a maintenance dose 150 or 220 mg once daily for 6-10 days is administered.

\section{Conclusion}

Having analyzed 6-year follow-up data, we can conclude that modern oral anticoagulant drugs are efficient and safe to apply in orthopaedics and traumatology. However, numerous studies show that these drugs should be used in outpatient period as well and cannot be used in short term only. Short-term inpatient prophylaxis fails to prevent thrombosis developing in outpatient period. In our opinion, the most efficient available drug is dabigatran, which demonstrated the best safety and efficacy levels among oral anticoagulants while being used in clinical practice in patients after large-scale orthopaedic surgeries.

\section{References}

[1] Prevention of Venous Thromboembolic Complications in Traumatology and Orthopedics. 2012. "Russian Clinical Recommendations." Travmatologiya i ortopediya Rossii 1 (63): 1-24. (in Russian)

[2] Botella Gabriel, F., Labios Gomez, M., Balaguer Martinez, J. V., and Fernández Llopez, A. 2009. "Prevention of Venous Thromboembolism: Unfractionated Heparins and Low-Molecular Weight Heparins. Analysis of These 2 Options.” An. Med. Interna. 16 (11): 590-600. (In Spanish)

[3] Camporese, G., Bernardi, E., Prandoni, P., Noventa, F., Verlato, F., and Simioni, P. 2008. "Low-Molecular-Weight Heparin Versus Compression Stocking for Thromboprohylaxis after Knee Arthroscopy: A Randomized Trial.” Ann. Intern Med. 149 (2): 73-82.

[4] Cohen, A. T., Agnelli, G., Anderson, F. A., Arcelus, J. I., Bergqvist, D., Brecht, J. G., Greer, I. A., Heit, J. A., Hutchinson, J. L., Kakkar, A. K., Mottier, D., Oger, E., Samama, M. M., and Spannagi, M. 2007. "VTE Impact
Assessment Group in Europe (VITAE). Venous thromboembolism (VTE) in Europe. The Number of VTE Events and Associated Morbidity and Mortality." Thromb Haemost 98 (4): 756-64.

[5] Carneiro, J. L., Targueta, G. P., and Marino, L. O. 2010. "Evalution of Venous Thromboembolism Prophylaxis in a High Complexity Hospital.” Rev. Col. Bras. Cir. 37 (3): 204-10.

[6] Dahl, O. E. 2008. "Efficacy and Safety Profile of Dabigatran Etexilate Compared with Enoxaparin in Primary Venous Thromboembolism Prevention after Total Knee or Hip Replacement Surgery in Patients over 75 Years." Blood 112: 167.

[7] Eikelboom, J. W., Quinlan, D. J., and Douketis, J. D. 2002. "Extended Duration Prophylaxis against Venous Thromboembolism after Total Hip or Knee Replacement: A Meta-Analysis of the Randomized Trials." Lancet 358: 9-15.

[8] Erikkson, B., Kakkar, A. K., Turpie, A. G., Gent, M., Bandel, T. J., Homering, M. et al. 2009. "Oral Rivaroxaban for the Prevention of Symptomatic Venous Thromboembolism after Elective Hip and Knee Replacement.” J. Bone Joint Surg. Br. 91 (5): 636-44.

[9] Hill, J., and Treasure, T. 2010. "Reducing the Risk of Venous Thromboembolism in Patients Admitted to Hospital: Summary of the NICE Guideline." Heart 96 (11): 879-82.

[10] NICE Clinical Guideline 46. 2007. "Venous Thromboembolism: Reducing the Risk of Venous Thromboembolism (Deep Vein Thrombosis and Embolism) in Inpatients Undergoing Surgery.” Available at www.guidance.nice.org.uk/CG46.

[11] Gussoni, G., Campanini, M., Silingardi, M., Scannapieco, G., Mazzone, A., Magni, G. et al. 2009. "In-Hospital Symptomatic Venous Thromboembolism and Antithrombotic Prophylaxis in Internal Medicine. Findings from a Multicenter, Prospective Study." Thromb. Haemost 101 (5): 893-901.

[12] Samama, C. M., Ravaud, P., Parent, F., Barré, J., Mertl, P., and Mismetti, P. 2007. "Epidemiology of Venous Thromboembolism after Lower Limb Arthroplasty: The FOTO Study." J. Thromb. Haemost 5 (12): 2360-7.

[13] Huisman, M. V., Quinlan, D. J., Dahl, O. E., and Schulman, S. 2010. "Enoxaparin versus Dabigatran or Rivaroxaban for Thromboprophylaxis after Hip or Knee Arthroplasty Results of Separate Pooled Analyses of Phase III Multicenter Randomized Trials." Cardiovasc. Qual. Outcomes 3 (6): 652-60.

[14] Rosecher, N. et al. 2012. "A New Approach with Anticoagulant Development: Tailoring Anticoagulant Therapy with Dabigatran Etexilate According to Patient Risk." Expert Opin. Pharmacother 13 (2): 217-26. 
[15] Turpie, A. G., Haas, S., Kreutz, R., Mantovani, L. G., Pattanayak, C. W., Holberg, G., Jamal, W., Schmidt, A. et al. 2014. "A Non-interventional Comparison of Rivaroxaban with Standard of Care for Thromboprophylaxis after Major Orthopaedic Surgery in 17,701 Patients with Propensity Score Adjustment." Thromb Haemost 111 (1): 94-102.

[16] Lassen, M. R., Gallus, A., Borris, L. C. et al. 2010. "Apixaban versus Enoxaparin for Thromboprophylaxis after Hip Replacement.” N. Engl. J. Med. 363: 2487-98.

[17] Geerts, W. H., Bergqvist, D., Pineo, G. F., Heit, J. A., Samama, C. M., Lassen, M. R., and Colwell, C. W. 2008. "Prevention of Venous Thromboembolism: American College of Chest Physicians Evidence-Based Clinical Practice Guidelines (8th Edition)." Chest 133 (6 Suppl): 381S-453S.

[18] Pellegrini Jr, V. D., Sharrock, N. E., Paiement, G. D., Morris, R., and Warwick, D. J. 2008. "Venous Thromboembolic Disease after Total Hip and Knee Arthroplasty: Current Perspectives in a Regulated Environment. AAOS Instr." Course Lect. 57: 637-61.

[19] Ramos, J., Perrotta, C., Badariotti, G., and Berenstein, G.
2007. "Interventions for Preventinng Venous Thromboembolism in Adults Undergoing Knee Arthroscopy." Cochrane Database Syst. Rev. 2: CD005259.

[20] Sandoval-Chagoya, G. A., and Laniado-Laborin, R. 2013. "Deep Vein Thrombosis Prophylaxis." Rev. Med. Inst. Mex. Seguro Soc. 51 (6): 688-91.

[21] Warwick, D., Dahl, O. E., and Fisher, W. D. 2008. "Orthopaedic Thromboprophylaxis: Limitations of Current Guidelines.” J. Bone Joint Surg. 90 (2): 127-32.

[22] Warwick, D., Friedman, R. J., Agnelli, G., Gil-Garay, E., Johnson, K., FitzGerald, G., and Turibio, F. M. 2007. "Insufficient Duration of Venous Thromboembolism Prophylaxis after Total Hip or Knee Replacement when Compared with the Time Course of Thromboembolic Events: Findings from the GLORY Global Orthopaedic Registry.” J. Bone Joint Surg. Br. 89 (6): 799-807.

[23] Gomez-Outes, A. 2012. "Dabigatran, Rivaroxaban, or Apixaban versus Enoxaparin for Thromboprophylaxis after Total Hip or Knee Replacement: Systematic Review, Meta-analysis, and Indirect Treatment Comparisons." BMJ 344: e36-e75. 